\section{A lasting marriage: histones and DNA tie a knot that is here to stay}

\author{
David Shechter and C. David Allis
}

DNA and protein have been together for a long time. Since the dawn of the DNA age, cells have had to handle information transactions with the long DNA poly-anion while preventing spurious electrostatic interactions and controlling its topology. The major eukaryotic innovation to tackle these problems has been the family of highly conserved and functional histone proteins.

In a typical eukaryotic cell, histones and DNA exist in a 1:1 mass ratio, and are organized into a fundamental repeating unit - the nucleosome - to form chromatin. Each nucleosome is composed of DNA wrapped around an octamer of the four core histones $\mathrm{H} 2 \mathrm{~A}, \mathrm{H} 2 \mathrm{~B}, \mathrm{H} 3$ and $\mathrm{H} 4$. This tight packaging, which is further helped by linker histones and other less-abundant proteins, produces condensed chromosomes that allow faithful segregation of the genetic material in dividing cells. Yet this close connection between histone and DNA is more than a marriage of convenience: histones are also crucial information carriers, containing epigenetic information that regulates the usage of the underlying DNA. This vision of the partnership took some time to emerge, however, as DNA held centre stage for several decades as the information bearer.

Histones were among the first proteins to be studied, as they were abundant and easily isolated. The electrostatic attraction that keeps DNA and histones together in the cell also provided early investigators with a simple technique to isolate both, thanks to their differential solubility in acid and base. Yet DNA, as a covalently linked polymer with only four discrete building blocks, was simpler to study than the dynamic histones. By the middle of the twentieth century, Avery and colleagues had demonstrated that DNA was the genetic material, and Watson and Crick had published its structure. As the present milestones collection illustrates, the DNA technologies that were then developed allowed the nascent field of molecular biology to galvanize the world with its ever-expanding insights into the biological secrets of the cell.

By contrast, the study of histones moved more slowly until the last decade, largely because the necessary technological tools were not available. Yet, in the half-century in which DNA technology overshadowed the analysis of histone proteins, many important technical advances were made. The acid and high-salt extractions of histones carried out by the Stedmans and others indicated that histone populations varied between cell types ${ }^{1}$. Allfrey and colleagues, utilizing metabolic labelling of cells, discovered abundant post-translational modification of histones, including acetylation, methylation and phosphorylation. These discoveries led the investigators to presciently propose that histone covalent modifications are active regulators of transcription ${ }^{2}$; this theory is now supported by genetic studies by Grunstein and others and by biochemical studies performed by Schreiber, Allis and others. At the same time, technological advances that were ideally suited to histone study were introduced, including the acid-urea gel of Panyim and Chalkley, which separates histone variants and differently modified histones ${ }^{3}$.

The more recent development of antibodies that specifically recognize post-translationally modified histones, pioneered by Turner and colleagues, has propelled the study of histones forward. Capitalizing on the existing techniques to extract and separate histones, the use of these antibodies in western blots has revealed many context-dependent histonemodification profiles. Their combination with genetic knockdown has shown the direct role of many gene products in fashioning physiologically relevant histone patterns. The chemical production of histone-tail peptides containing one or more modifications has fuelled antibody production, and has, in turn, been used to identify proteins (or 'readers') that bind specific histone marks.

To further explore the complex world of the specific modifications exposed by histone antibodies, researchers have now turned to the expanding catalogue of massspectroscopy techniques. In addition to identifying post-translational modifications of histones, mass spectrometry has led to increased understanding of the linkage between particular modifications on the same polypeptide. Enzyme systems responsible for maintaining the steady-state balance of these marks ('writers' and 'erasers') continue to be identified.

So, thanks to technological developments that have happened independently, DNA and histones have both been revealed to play important roles as carriers of information. Now, the ultimate question is how to correlate histone epigenetic information with its corresponding DNA loci - the physical links of their 'marriage'. Elucidating this fundamental aspect of the relationship between DNA and histones requires the optimal combination of technologies that were developed to study one or the other.

The technique of chromatin immunoprecipitation (ChIP), in its various forms ${ }^{4}$, has proven to be a technological platform that is particularly amenable to combining histone and DNA methods. Over time, ChIP using modification-specific histone antibodies has been combined with a panoply of DNA technologies: nucleic-acid hybridization, realtime PCR, microarrays and, most recently, high-throughput DNA sequencing. These technological achievements have permitted the genetic localization of particular modifications and histone variants. The latest approach, nicknamed ChIP-Seq, makes possible rapid and high-resolution 'epigenomic' studies ${ }^{5}$. With further developments to increase the reliability of the antibodies and the expanding DNA-sequencing technologies, ChIP-Seq techniques are likely to be crucial in understanding epigenetic information flow and, perhaps eventually, in clinical diagnosis. At last, the technology seems to be in place to directly explore the intimate relationship between histones and DNA. Despite having gone their own way for several decades, histone proteins and DNA are back together in the focus of researchers. Their marriage has proven to be a long-lasting one.

David Shechter and C. David Allis are at The Laboratory of Chromatin Biology, The Rockefeller University, 1230 York Avenue, New York, New York 10065, USA.

e-mail: alliscd@rockefeller.edu doi: $10.1038 / \mathrm{nrg} 2249$

Stedman, E. \& Stedman, E. Cell specificity of histones. Nature 166, 780-781 (1950).

2. Allfrey, V. G., Faulkner, R. \& Mirsky, A. E. Acetylation and methylation of histones and their possible role in the regulation of RNA synthesis. Proc. Natl Acad. Sci. USA 51, 786-794 (1964)

3. Panyim, S. \& Chalkley, R. High resolution acrylamide gel electrophoresis of histones. Arch. Biochem. Biophys. 130, 337-346 (1969).

4. Solomon, M. J., Larsen, P. L. \& Varshavsky, A. Mapping protein-DNA interactions in vivo with formaldehyde: evidence that histone $\mathrm{H} 4$ is retained on a highly transcribed gene. Cell 53, 937-947 (1988).

Barski, A. et al. High-resolution profiling of histone methylations in the human genome. Cell 129 , 823-837 (2007). 